\title{
Ritidoplastia em face espástica: relato de caso
}

\author{
Rhytidoplasty in patient with hemifacial spasm: case report
}

\author{
Allysson Antonio Ribeiro \\ Gomes $^{1}$ \\ Salustiano Gomes de \\ Pinho Pessoa ${ }^{2}$ \\ Everardo Macedo ${ }^{3}$ \\ Nelson Gurgel Simas de \\ Oliveira $^{1}$ \\ IANa Silva Dias ${ }^{1}$
}

Trabalho realizado no Hospital Universitário Walter Cantídio -

Universidade Federal do Ceará

Fortaleza, CE, Brasil.

Artigo submetido pelo SGP (Sistema de Gestão de Publicações) da RBCP.

Artigo recebido: 28/9/2009

Artigo aceito: 6/4/2010

\begin{abstract}
RESUMO
Introdução: O espasmo hemifacial (EH) é um distúrbio do movimento caracterizado por contrações involuntárias dos músculos inervados pelo nervo facial, a descompressão microvascular (DMV) é considerada tratamento definitivo, com baixas taxas de recidiva. Os casos refratários tornam-se um desafio para o cirurgião plástico. Relato do Caso: MSP, sexo feminino, 53 anos, portadora de $\mathrm{EH}$, refratária à DMV, e com queixas de flacidez dos dois terços inferiores da face. Foi submetida à ritidoplastia, neurectomias e miectomias seletivas. Obteve-se ausência completa dos espasmos, manutenção da mímica facial e rejuvenescimento facial. A ritidoplastia cérvico-facial associada a neurectomias e miectomias mostrou-se eficaz no tratamento do EH.
\end{abstract}

Descritores: Face/cirurgia. Espasmo hemifacial. Espasmo. Ritidoplastia.

\begin{abstract}
Introduction: The peripheral facial palsy is a disease that can leave significant aesthetical and functional sequelae in patients. According to medical literature, Bell's palsy (BP) is the most common cause, representing from 50 to $80 \%$ of the cases. However, it must be considered as an exclusion diagnosis. A slightly higher prevalence is estimated in women and its incidence is bimodal with peaks in the third and eighth life decades. Most patients recover within weeks, though a worsening degree may be common in the first 48 hours. Treatment must be performed to prevent complications and the medical procedures are not universally accepted. The following have been mentioned as BP risk factors: arterial hypertension, mellitus diabetes, pregnancy, puerperium and infection caused by type I herpes virus. Although there are several BP associated risk factors, regarding face-lifting surgery there is only one reported case in medical literature. Case Report: In the present study, the authors describe the second case of Bell's palsy after face-lifting surgery.
\end{abstract}

Keywords: Face/surgery. Hemifacial spasm. Spasm. Rhytidoplasty.

1. Pós-graduação em cirurgia geral; Residente do Serviço de Cirurgia Plástica e Microcirurgia Reconstrutiva Credenciado pela Sociedade Brasileira de Cirurgia Plástica (SBCP) e MEC Hospital Universitário Walter Cantídio - Universidade Federal do Ceará, Fortaleza, CE, Brasil.

2. Membro Titular da SBCP, Regente do Serviço de Cirurgia Plástica e Microcirurgia Reconstrutiva Hospital Universitário Walter Cantídio - Universidade Federal do Ceará São Lucas - Hospital de Cirurgia e Anestesia, Fortaleza, CE, Brasil.

3. Membro Titular da SBCP, Preceptor do Serviço de Cirurgia Plástica e Microcirurgia Reconstrutiva Hospital Universitário Walter Cantídio - Universidade Federal do Ceará, Fortaleza, CE, Brasil. 


\section{INTRODUÇÃO}

O espasmo hemifacial (EH) é um distúrbio do movimento caracterizado por contrações involuntárias paroxísticas dos músculos inervados pelo nervo facial ${ }^{1}$. A forma de apresentação mais comum é a contração involuntária da metade inferior da musculatura orbicular dos olhos, unilateralmente, que piora de frequência e intensidade, seguindo-se caudalmente, podendo acometer o músculo platisma em $15 \%$ dos casos ${ }^{2}$.

A etiopatogenia que tem sido proposta para o EH é a compressão do VII par craniano ${ }^{3}$ por malformações vasculares $^{4}$ ou tumores (lipomas ${ }^{5}$, meningiomas ${ }^{6}$ ) localizados no ângulo pontocerebelar da fossa posterior do crânio. É uma doença subdiagnosticada, mas se sabe que acomete predominantemente o sexo feminino, em proporção de $3: 1^{2,7}$, com idade média de incidência de 55 anos $^{8}$, e prevalência total variando entre 9,8 a 11 por 100.000 habitantes em populações orientais.

Injeções de toxina botulínica, para promover quimiodesnervação da musculatura acometida, são amplamente aceitas para o tratamento de casos de $\mathrm{EH}$ precoces ${ }^{8}$, e a descompressão microvascular (DMV) do nervo facial é considerado tratamento definitivo, com baixas taxas de recidiva ${ }^{9}$. A redução da atividade muscular da face também pode ser obtida por neurotomias seletivas e miectomias ${ }^{10}$.

A preocupação com aparência é comum em nosso meio, e a ritidoplastia é a cirurgia com a finalidade de rejuvenescimento que se ocupa de diminuir os sinais do envelhecimento produzido pela ação muscular e pela gravidade, além de proporcionar acesso cirúrgico para realização de neurotomias e miectomias.

O presente estudo tem por objetivo relatar um caso de tratamento cirúrgico com ritidoplastia associada a neurectomias e miectomias em paciente portador de EH refratário à DMV.

\section{RELATO DE CASO}

MSP, 53 anos, sexo feminino, com quadro de contrações paroxísticas involuntárias hemifaciais à esquerda envolvendo musculatura peri-orbicular, terço médio da face e platisma, no território de inervação do nervo facial. Queixa de flacidez terço médio da face e região mandibular. Negava comorbidades associadas, tabagismo e/ou alergias. Realizou quimiodesnervação com toxina botulínica, apresentando progressivo aumento da frequência de utilização e redução do intervalo entre as aplicações. Foi submetida à DMV, apresentando recidiva dos sintomas. $\mathrm{O}$ exame físico demonstrou: contrações clônicas dos músculos da hemiface esquerda; elevação da cauda do supercílio esquerdo, com assimetria acentuada devido à contração do músculo frontal esquerdo; canto lateral da pálpebra esquerda elevado; sulcos nasogenianos profundos, sendo o esquerdo mais evidente; brida platismal esquerda mais evidente que a direita; músculo platisma tipo III de Cardoso de Castro $^{11}$; apagamento da mandíbula bilateralmente (Figura 1).

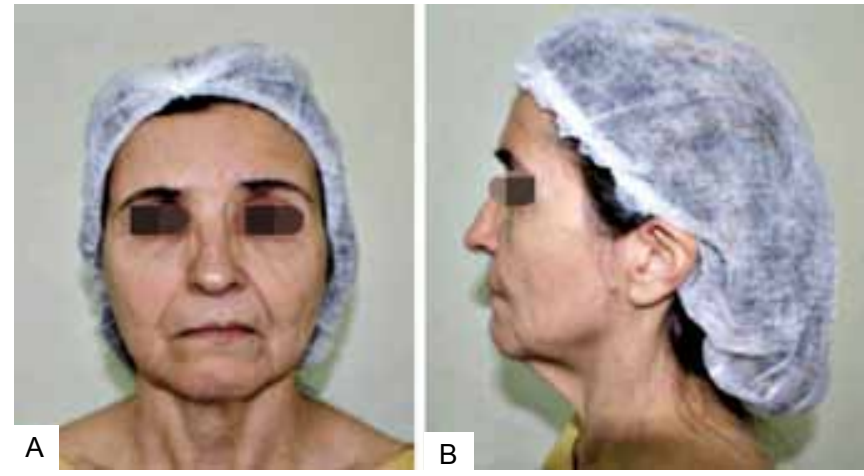

Figura 1 - Pré-operatório. A: visão frontal; B: perfil esquerdo.

Após o termo de consentimento pós-informado sobre a proposta cirúrgica, possíveis complicações e autorização para publicação de fotografias com finalidade científica ter sido lido e aceito pela paciente e testemunhas, a mesma foi submetida a ritidoplastia cérvico-facial, miectomias e neurectomias.

\section{Técnica cirúrgica}

A paciente, sob anestesia local e sedação, foi submetida a incisão pré-pilosa, tricofítica, pré-tragal, contornando o pavilhão auricular e seguindo em semicírculo com concavidade inferior até atingir a linha de implantação do cabelo. O descolamento em plano superficial, contornando a orelha com prolongamentos superior, anterior, inferior e posterior, proporcionou amplo acesso à região periorbitária látero-inferior, assim como à região malar e platisma.

Utilizando-se eletroestimulador, foram localizados os ramos temporal e zigomático do VII nervo craniano, e seguiuse com a realização de neurectomias de aproximadamente 1 $\mathrm{cm}$ daqueles ramos que causavam as contrações da região periorbitária esquerda. O mesmo foi realizado para identificar ramos bucais e realizar neurectomias seletivas para tratar a região geniana.

Foi realizada miectomia látero-inferior do terço lateral do músculo orbicular pré-septal e sua porção orbitária, com $3 \mathrm{~cm}$ de extensão. Procedeu-se à miectomia do platisma, removendo uma faixa de $3 \mathrm{~cm}$ de largura na região submandibular esquerda, atingindo todo o comprimento do músculo em sua inserção na mandíbula, preservando o nervo marginal da mandíbula e realizando acesso cirúrgico submentoniano para auxiliar a remoção da brida platismal na região cervical anterior (Figura 2).

Seguiu-se à ressecção de pele, obedecendo aos vetores de tração usuais da ritidoplastia, lipoenxertia nos locais de miectomia com gordura do excesso de pele removido, sutura por planos e drenagem a vácuo e curativo em forma de capacete. 

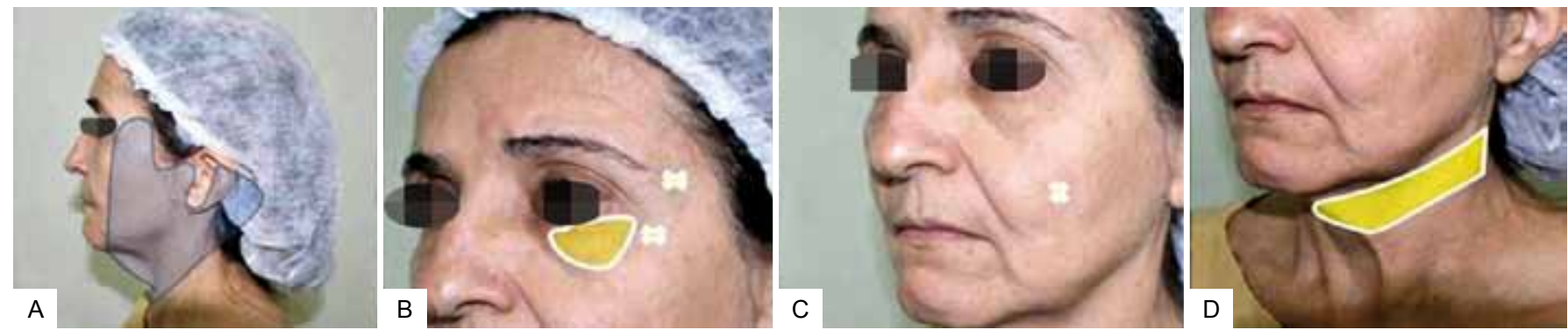

Figura 2 -Planejamento cirúrgico. A: descolamento do retalho cutâneo; B: miectomia do músculo orbicular (área em amarelo) e neurectomias de ramos temporal e zigomático; $\boldsymbol{C}$ : neurectomia de ramos bucinadores; D: miectomia do platisma.

O mesmo procedimento foi realizado em hemiface direita, exceto as neurectomias e miectomias.

A paciente permaneceu assintomática por 24 horas em observação, em regime hospitalar, recebendo alta da enfermaria após remoção do sistema de drenagem bilateral e troca de curativo. Observou-se ausência de contrações involuntárias em região peri-orbitária esquerda, da musculatura da região malar e do platisma. Simetrização das sobrancelhas, abaixamento da cauda do supercílio, atenuação do sulco nasogeniano esquerdo. Melhora do contorno da mandíbula e do queixo. As cicatrizes apresentaram boa qualidade e sem estigmas.

Obteve-se ausência completa dos espasmos, manutenção da mímica facial com ação do músculo frontal e do músculo orbicular remanescente, com oclusão palpebral adequada. O resultado do procedimento realizado mantém inalterado durante os dois anos de seguimento pós-operatório, demonstrando o controle duradouro da doença. Além disso, proporcionou correção das deformidades secundárias à ação da contratilidade muscular e da gravidade (Figura 3).

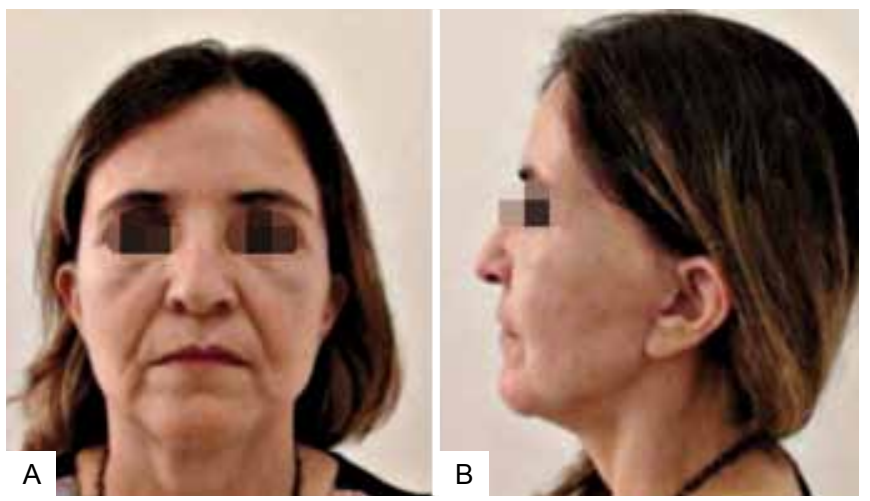

Figura 3 - Pós-operatório. A: visão frontal; B: perfil esquerdo.

\section{DISCUSSÃO}

Embora descrito há mais de um século, o EH continua sendo uma entidade nosológica subdiagnosticada ou confundida com seus principais diagnósticos diferenciais: blefaroespasmo essencial, miokimia ou síndrome de Gilles de La Tourette.

Usualmente, o quadro clínico inicia-se na musculatura orbicular da pálpebra e espalha-se em direção caudal, acometendo a musculatura da mímica facial e menos frequentemente atinge o músculo platisma ${ }^{2,6}$ como no caso descrito.

Como tratamento primordial para o $\mathrm{EH}$, é unânime a opção pela injeção de toxina botulínica, devido à segurança e à eficácia. Injetada em subcutâneo ou intramuscularmente nas regiões acometidas, tendo início de ação em torno de 3 a 5 dias, com duração média de 3 a 6 meses. Entretanto, o paciente necessita de repetidas aplicações por ano, o que pode ser economicamente inviável para casos de extenso acometimento ${ }^{5,8,12}$ e para aqueles que apresentam diminuição na resposta terapêutica, tendo-se necessidade de optar pela complementação cirúrgica da resolução do problema.

Baseado no recente entendimento do mecanismo fisiopatológico do EH, a DMV é considerada curativa para aqueles pacientes com diagnóstico de compressão vascular do VII nervo através de angioressonância magnética, sendo a artéria cerebelar ântero-inferior a mais comumente envolvida, e apresentando baixos índices de recidiva após um ano de tratamento, como foi evidenciado por Yuan et al. ${ }^{9}$, em 2005, estudando 1200 casos operados, e com 2 a 10 anos de seguimento, com taxa de ineficácia em 2,6\%, recorrência de $3,2 \%{ }^{4,11}$. O baixo índice de recidivas após a DMV torna os casos refratários desafiadores.

Em 1967, Reynolds, Smith e Walsh descreveram a neurectomia de Reynolds, que consiste em excisão periférica do nervo facial, que apresenta graves efeitos colaterais como paresia facial, além ectrópio paralítico e ptose de supercílio $^{7}$. Muhlbauer et al. ${ }^{13}$ publicaram, em 1995, a 
modulação mimética, relatando miectomias do músculo orbicular, e neurotomias seletivas dos ramos motores para esta musculatura, com finalidade estética de correção de rugas dinâmicas neste local. Em 2000, Bonatto et al. ${ }^{10}$ descreveram abordagem transpalpebral para miectomias, com resultados satisfatórios.

Utilizando eletroestimulador, foi possível identificar com precisão os ramos dos nervos temporal e zigomático, e ramos dos nervos bucais que eram responsáveis pelos espasmos periorbiculares e da região geniana, respectivamente. As neurectomias seletivas, realizadas por meio de incisão de ritidoplastia, restritas às áreas acometidas, permitiram evitar as complicações descritas por Reynolds ${ }^{10}$. A realização de lipoenxertia do tecido adiposo retirado do SMAS evita depressão no local da miectomia do orbicular.

As intervenções sobre o músculo platisma tiveram grande impulso na década de 70, Connell \& Marten $^{14}$, em 1978, propuseram secções no músculo platisma que podiam ser totais ou parciais, e tração posterior, promovendo excelentes resultados no que diz respeito à delineação da mandíbula. Esta técnica foi utilizada realizando-se duplo acesso: ritidoplastia e na prega submentoniana; sendo removida uma faixa de músculo platisma e neurectomia do ramo cervical do nervo facial, uma vez que não é possível a dissecção desde nervo da musculatura efetora. Conseguiu-se o controle do EH, e rejuvenescimento da região cervical.

\section{REFERÊNCIAS}

1. Jannetta PJ. Hemifacial spasm. In: Samii M, Jannetta PJ, eds. The cranial nerves. New York:Springer- Verlag;1981. p.484-94.

2. Barbosa ER, Costa MDL, Staut CC, Bacheschi LA, Bittar MS. Espasmo hemifacial familiar: relato de dois casos. Arq Neuro-Psiquiatr. 1998;56(1):111-5.

3. Campbell E, Keedy C. Hemifacial spasm; a note on the etiology in two cases. J Neurosurg. 1947;4(4):342-7.

4. Jannetta PJ, Kassam A. Hemifacial spasm. J Neurol Neurosurg Psychiatry. 1999;66(2)255-6.

5. Fagundes-Pereyra WJ, Marques JAP, Carvalho GTC, Sousa AA. Lipoma do ângulo pontocerebelar. Arq Neuro-Psiquiatr. 2000;58(3B):952-7.

6. Cancelli I, Cecotti L, Valentinis L, Bergonzi P, Gigli GL. Hemifacial spasm due to a tentorial paramedian meningioma: a case report. Neurol Sci. $2005 ; 26(1): 46-9$.

7. Friedhofer H, Oliveira RR, Paula PRS, Limongi JCP, Ferreira MC. Tratamento cirúrgico do blefaroespasmo essencial: relato de dois pacientes. Arq. Neuro-Psiquiatr. 1999;57(2B):476-83.

8. Miwa H, Mizuno Y, Kondo T. Familial hemifacial spasm: report of cases and review of literature. J Neurol Sci. 2002;193(2):97-102.

9. Yuan Y, Wang Y, Zhang SX, Zhang L, Li R, Guo J. Microvascular decompression in patients with hemifacial spasm: report of 1200 cases. Chin Med J (Engl). 2005;118(10):833-6.

10. Bonato A, Viterbo F, Melega JM. Neurotomias e miectomias. In: Melega JM, ed. Cirurgia Plástica Fundamentos e Arte. São Paulo:Editora Guanabara;2003. p.258-70.

11. Cardoso de Castro C. The anatomy of the platysma muscle. Plast Reconstr Surg. 1980;66(5):680-3

12. Kong DS, Park K. Hemifacial spasm: a neurosurgical perspective. J Korean Neurosurg Soc. 2007;42(5):355-62.

13. Muhlbauer W, Fairley J, van Wingerden J. Mimetic modulation for problem creases of the face. Aesthetic Plast Surg. 1995;19(2):183-91.

14. Connell BC, Marten TJ. The value of platysma muscle flaps. Ann Plast Surg. 1978;1:34-43.

\section{Correspondência para:}

Allysson Antonio Ribeiro Gomes

Rua Thomas Pompeu, 550 - apto. 203 - Meireles - Fortaleza, CE, Brasil - CEP 60160-080

E-mail: dr.allyssongomes@hotmail.com 